\title{
LOCALIZED AND COMPUTATIONALLY EFFICIENT APPROACH TO SHIFT-VARIANT IMAGE DEBLURRING
}

\author{
Murali Subbarao, Youn-sik Kang, Satyaki Dutta*, Xue Tu \\ \{murali, yskang, tuxue\}@ece.sunysb.edu, sunny@math.sunysb.edu \\ Dept. of Electrical and Computer Engg., State University of New York at Stony Brook, NY 11794 \\ *Dept. of Mathematics, SUNY at Stony Brook, NY 11794
}

\begin{abstract}
A new localized and computationally efficient approach is presented for shift/space-variant image restoration. Unlike conventional approaches, it models shift-variant blurring in a completely local form based on the recently proposed Rao Transform (RT). RT facilitates almost exact inversion of the blurring process locally and permits very fine-grain parallel implementation. The new approach naturally exploits the spatial locality of blurring kernels and smoothness of underlying focused images. It formulates the deblurring problem in terms of local parameters that are less correlated than raw image data. It is a fundamental advance that is general and not limited to any specific form of the blurring kernel such as a Gaussian. It has significant theoretical and computational advantages in comparison with conventional approaches such as those based on Singular Value Decomposition of blurring kernel matrices. Experimental results are presented for both synthetic and real image data. This approach is also relevant to solving integral equations.
\end{abstract}

Index Terms - Shift/Space-Variant Image Restoration, Deblurring, Deconvolution, Integral Equations

\section{INTRODUCTION}

Restoration of shift/space-variant blurred images to recover focused images is an important problem in digital imaging and machine vision $[1,2,3,4,5,6,7]$. Such blur may be due to 3D shape or motion of objects in digital cameras/microscopes, optical aberrations in imaging systems, or atmospheric turbulence in astronomical telescopes. Many approaches have been proposed in the literature for restoring such images to recover the underlying focused image. For example, see $[1,4,5,6,7]$ and the references there.

Conventional approaches to modeling shift-variant or space-variant blurring of images is not fully localized and computationally exorbitant. Typically, a large shift-variant blurred image is divided into many smaller image blocks of size $K \times K$ where $K$ is around 32 or 64 and restored separately and then synthesized. A two-dimensional image block is converted to a very large $K^{2} \times 1$ one-dimensional vector b by rearranging the columns of the image block vertically one below another. The shift-variant blurring kernel or point spread function (SV-PSF) is specified by a huge $K^{2} \times K^{2}$ matrix $\mathbf{A}$. The focused image vector $\mathbf{x}$, again represented as a large $K^{2} \times 1$ column vector, is obtained by solving the matrix equation $\mathbf{A x}=\mathbf{b}$ which has a very high computational complexity of $O\left(K^{6}\right)$. Unlike the new approach proposed here, this formulation of the problem does not exploit the natural locality and limited support domain of the physical blurring kernel and spatial smoothness of focused images.

A spatial-domain convolution/deconvolution transform (S Transform) was proposed and demonstrated for modeling and restoring shift-invariant (i.e. convolution) blurred images [2, 3]. Recently, this approach has been extended to shift-variant image deblurring $[8,9]$ based on a new transform named Rao Transform (RT). This new approach models the blurring process in a completely local form that naturally exploits the spatial locality and limited support domain of blurring kernels (SV-PSFs). Further, it formulates the problem in terms of local parameters (e.g. derivatives) of an analytic approximation to the focused image. These parameters are much more independent and uncorrelated with each other than raw pixel data used in conventional methods. Thus it exploits spatial smoothness in images. As a consequence, in a typical practical application, the new approach reduces computation significantly, and provides a new theory. In this sense, the new approach represents a fundamental theoretical and computational advance. Further, RT is also relevant to solving integral/differential equations and shape from defocus $[8,9]$. First we present the computational algorithm and analysis of the new approach and then the experimental results.

\section{RT THEORY, ALGORITHM, AND ANALYSIS}

In the continuous domain, the shift-variant point spread function (SV-PSF), the focused image, and the corresponding blurred image are represented by $h(x, y, u, v), f(x, y)$, and $g(x, y)$ respectively. The conventional continuous domain blurring model uses the global form SV-PSF $k$, and is given by

$$
g(x, y)=\int_{a}^{b} \int_{c}^{d} k(x, y, u, v) f(u, v) d u d v
$$


A localized kernel $h(x, y, u, v)$ corresponding to $k(x, y, u, v)$ can be defined as [8]

$$
h(x, y, u, v)=k(x+u, y+v, x, y)
$$

A completely localized blurring model which is exactly equivalent to Eq. (1) can be obtained by the change of variables $u^{\prime}=x-u$ and $v^{\prime}=y-v$. The resulting expression defines Rao Transform (RT):

$g(x, y)=\int_{x-b}^{x-a} \int_{y-d}^{y-c} h(x-u, y-v, u, v) f(x-u, y-v) d u d v$

Next we derive the inverse of RT to solve the above integral equation for the focused image $f$. The $m^{\text {th }}$ partial derivative with respect $x$ and the $n^{\text {th }}$ partial derivative with respect to $y$ of a function will be denoted by the superscript $(m, n)$ for the function, for example, $g^{(m, n)}, f^{(m, n)}$, and $h^{(m, n)}$. The $(p, q)^{t h}$ moments with respect to $u, v$ of $h^{(m, n)}$ are defined by

$$
\begin{aligned}
h_{p, q}^{(m, n)} & =h_{p, q}^{(m, n)}(x, y) \\
& =\int_{x-b}^{x-a} \int_{y-d}^{y-c} u^{p} v^{q} h^{(m, n)}(x, y, u, v) d u d v
\end{aligned}
$$

for $m, n, p, q=0,1,2, \ldots$ Note that, for all SV-PSFs, by definition (follows from the conservation of light energy),

$$
h_{0,0}^{(0,0)}(x, y)=\int_{-\infty}^{\infty} \int_{-\infty}^{\infty} h(x, y, u, v) d u d v=1
$$

for all $(x, y)$ and therefore, all derivatives of $h_{0,0}^{(0,0)}$ with respect to $x$ and $y$ are zero. Also, although unnecessary for a theoretical development of the method, due to its practical utility, we shall assume that $h$ is symmetric with respect to $u$ and $v$, i.e. $h(x, y, u, v)=h(x, y,|u|,|v|)$, which is the case for 2D Gaussian, cylindrical and rect functions. In this case, derivatives with respect to $x$ and $y$ are also symmetric, i.e.

$$
h^{(m, n)}(x, y, u, v)=h^{(m, n)}(x, y,|u|,|v|)
$$

Using the relations $u^{p} v^{q}=-(-u)^{p} v^{q}$ when $p$ is odd and $u^{p} v^{q}=-u^{p}(-v)^{q}$ when $q$ is odd, we find that $h_{p, q}^{(m, n)}(x, y)=$ 0 when $p$ is odd or $q$ is odd.

Using the above notation, the truncated Taylor series expansion of $f(x-u, y-v)$ around $(x, y)$ up to order $N$ and $h(x-u, y-v, u, v)$ around the point $(x, y, u, v)$ up to order $M$ will be used below. For example, we express

$$
h(x-u, y-v, u, v) \approx \sum_{m=0}^{M} a_{m} \sum_{j=0}^{m} C_{j}^{m} u^{m-j} v^{j} h^{(m-j, j)}
$$

where $C_{i}^{n}$ and $C_{j}^{m}$ denote the binomial coefficients and $a_{m}=$ $(-1)^{m} / m$ !. Substituting the truncated Taylor-series expansions of $h$ and $f$ into the RT in Eq. (3) and simplifying, we get

$$
g(x, y) \approx \sum_{n=0}^{N} a_{n} \sum_{i=0}^{n} C_{i}^{n} f^{(n-i, i)} \sum_{m=0}^{M} a_{m} \sum_{j=0}^{m} C_{j}^{m} h_{m+n-i-j, i+j}^{(m-j, j)}
$$

The above equation can be rewritten as

$$
g(x, y) \approx \sum_{n=0}^{N} \sum_{i=0}^{n} S_{n, i} f^{(n-i, i)}
$$

where

$$
S_{n, i}=a_{n} C_{i}^{n} \sum_{m=0}^{M} a_{m} \sum_{j=0}^{m} C_{j}^{m} h_{m+n-i-j, i+j}^{(m-j, j)}
$$

Equation (9) above is in a completely localized form in the sense that it expresses the blurred image $g$ at $(x, y)$ in terms of the derivatives of the focused image $f$ and the moments of the blurring kernel $h$ at the same point $(x, y)$. This is a basic new result that facilitates inversion of the blurring process exactly at the point $(x, y)$.

We can now write expressions for the various partial derivatives of order $(p, q)$ of $g$ with respect to $x, y$, as

$$
g^{(p, q)} \approx \sum_{n=0}^{N} \sum_{i=0}^{n} \frac{\partial^{p}}{\partial x^{p}} \frac{\partial^{q}}{\partial y^{q}}\left[S_{n, i} f^{(n-i, i)}\right] T(n+p+q)
$$

where

$$
T(n+p+q)= \begin{cases}1 & \text { if } n+p+q \leq N \\ 0 & \text { otherwise }\end{cases}
$$

assures that terms with derivatives of $f$ of order greater than $N$ are set to zero, for $p+q=0,1,2, \ldots, N$. Note that

$$
\begin{aligned}
S_{n, i}^{(p, q)} & =\frac{\partial^{p}}{\partial x^{p}} \frac{\partial^{q}}{\partial y^{q}} S_{n, i} \\
& =a_{n} C_{i}^{n} \sum_{m=0}^{M-(p+q)} a_{m} \sum_{j=0}^{m} C_{j}^{m} h_{m+n-i-j, i+j}^{(m-j+p, j+q)}
\end{aligned}
$$

The above equation for $g^{(p, q)}$ for $p, q=0,1,2, \ldots, N$, and $0 \leq p+q \leq N$ constitute $(N+1)(N+2) / 2$ equations in as many unknowns $f^{(p, q)}$. The system of equations for $g^{(p, q)}$ can be expressed in a vector-matrix form as

$$
\left[\begin{array}{c}
g^{(0,0)} \\
g^{(1,0)} \\
\vdots \\
g^{(0, N)}
\end{array}\right]=\left[\begin{array}{cccc}
r_{00} & r_{01} & \cdots & \cdots \\
r_{10} & r_{11} & \cdots & \cdots \\
\vdots & \vdots & \ddots & \vdots \\
& \cdots & &
\end{array}\right]\left[\begin{array}{c}
f^{(0,0)} \\
f^{(1,0)} \\
\vdots \\
f^{(0, N)}
\end{array}\right]
$$

or

$$
\mathbf{g}_{x, y}=\mathbf{R}_{x, y} \mathbf{f}_{x, y}
$$


where the subscripts $(x, y)$ make explicit the dependence of the vectors/matrix on $(x, y) . \mathbf{R}_{x, y}$ is the RT coefficient matrix of size $(N+1)(N+2) / 2$ rows and columns. This matrix equation can be solved to obtain $f^{(p, q)}$, and in particular, $f^{(0,0)}$ by inverting the kernel matrix $\mathbf{R}_{x, y}$. The solution can be written in the form

$$
\mathbf{f}_{x, y}=\mathbf{R}_{x, y}^{\prime} \mathbf{g}_{x, y}
$$

where $\mathbf{R}_{x, y}^{\prime}=\mathbf{R}_{x, y}^{-1}$ and the solution for $f^{(0,0)}$ can be expressed as

$$
f(x, y)=f^{(0,0)}=\sum_{n=0}^{N} \sum_{i=0}^{n} S_{n, i}^{\prime} g^{(n-i, i)}
$$

where the coefficient $S_{n, i}^{\prime}$ is obtained by a suitable grouping of the terms. The solution above needs to be computed at each pixel $(x, y)$. As estimating accurate image derivatives is difficult in practice, a regularization approach such as the Truncated Singular Value Decomposition (TSVD) [4] or Tikhonov regularization can be used to solve Eq. (15) above to obtain a smooth and stable solution for $f$.

In practice we find that the truncation of the Taylor series expansion of the kernel $h$ is useful even for small values of $M$, typically $M=1$ or at most 2 . Error introduced by the truncation of the Taylor series of an analytic approximation to the focused image $f$ depends on two factors: image noise and size of significant region of blurring kernel $h$. Quantization noise in 8 bits/pixel images usually limits the use of image derivatives to an order of $N=2$ or $N=3$ to be the maximum. The significant region of blurring kernel $h$ is roughly equal to the size of the maximum blur circle, which can be specified roughly by

$$
h(x-u, y-v, u, v) \approx 0 \text { for } u^{2}+v^{2}>R^{2}
$$

where $R$ could be the radius of the maximum blur circle size or $2 \sigma$ for a Gaussian kernel. The useful maximum value of $R$ is typically limited by the value of $N$, because, within the region of significant support of the SV-PSF, the truncated Taylor series expansion of the focused image should be a good approximation to the actual focused image. In summary, in practical applications with 8 bits/pixel, $N$ is limited to be around 3 due to image noise, which in turn limits the maximum blur circle size $R$ to yield acceptable errors in restoration. In our experiments, we find that for an 8 bits per pixel image, a value of $N=2$ in turn limits the maximum blur circle diameter to be around 7 pixels. This is still a very useful and practical approach that provides good restoration results and new insights into the nature and structure of the shiftvariant deblurring problem.

\subsection{Example}

We present a solution for the case of $N=2, M=1, a=$ $c=-\infty$, and $b=d=\infty$, for the case of a 2-D SV-PSF. In this case, Eq. (9) becomes

$$
\begin{aligned}
g^{(0,0)} & =f^{(0,0)}+f^{(1,0)} h_{2,0}^{(1,0)}+f^{(0,1)} h_{0,2}^{(0,1)} \\
& +\frac{1}{2} f^{(2,0)} h_{2,0}^{(0,0)}+\frac{1}{2} f^{(0,2)} h_{0,2}^{(0,0)}
\end{aligned}
$$

The above equation gives a method of computing the output blurred image $g(x, y)$ given the input focused image $f(x, y)$. This equation could be used in Computer Graphics for rendering a realistic image generated by a camera with limited depth of field. Eq. (17) gives the following completely localized explicit solution for $f^{(0,0)}$ at a point $(x, y)$ in terms of the derivatives of $g$ and moments of the derivatives of $h$ at the same point $(x, y)$ as below

$$
\begin{aligned}
f^{(0,0)} & =g^{(0,0)}-g^{(1,0)} h_{2,0}^{(1,0)}-g^{(0,1)} h_{0,2}^{(0,1)} \\
& +g^{(2,0)}\left(\frac{3}{2}\left(h_{2,0}^{(1,0)}\right)^{2}+\frac{1}{2} h_{0,2}^{(0,1)} h_{2,0}^{(0,1)}-\frac{1}{2} h_{2,0}^{(0,0)}\right) \\
& +g^{(0,2)}\left(\frac{3}{2}\left(h_{0,2}^{(0,1)}\right)^{2}+\frac{1}{2} h_{2,0}^{(1,0)} h_{0,2}^{(1,0)}-\frac{1}{2} h_{0,2}^{(0,0)}\right)
\end{aligned}
$$

Further simplification of the above equation is possible when the kernel is rotationally symmetric (e.g. $h_{2,0}^{(0,0)}=h_{0,2}^{(0,0)}$ and therefore $h_{2,0}^{(p, q)}=h_{0,2}^{(p, q)}$ ). The above equation gives an explicit, closed-form formula for restoring an image blurred by a shift-variant symmetric point spread function. Such a closedform solution is new and represents a basic theoretical advance. Closed-form expressions for $h_{p, q}^{(m, n)}(x, y)$ in the case of a 2D Gaussian is given below. Similar closed form expressions can be obtained for Cylindrical and rect SV-PSFs [8].

The Gaussian SV-PSF and its moments are:

$$
\begin{gathered}
h(x, y, u, v)=\frac{1}{2 \pi \sigma^{2}(x, y)} \exp -\left(\frac{u^{2}+v^{2}}{2 \sigma^{2}(x, y)}\right) \\
h_{p, q}(x, y)=\left\{\begin{array}{l}
0 \quad \text { if } p \text { is odd OR } q \text { is odd } \\
\frac{(\sigma \sqrt{2})^{p+q}}{4 \pi} \Gamma\left(\frac{p+1}{2}\right) \Gamma\left(\frac{q+1}{2}\right) \text { otherwise }
\end{array}\right.
\end{gathered}
$$

$h_{p, q}^{(m, n)}(x, y)$ are obtained by differentiating the above.

\subsection{Computational Complexity}

The computational needs of a conventional method (e.g. TSVD) is $O\left(K^{6}\right)$ for an image of size $K \times K$. In comparison, the computational complexity of the RT approach is $O\left(K^{2} N^{6}\right)$ because the computations are dominated by the inversion of $\mathbf{R}_{x, y}$ of size $O\left(N^{2}\right)$ at $K^{2}$ pixels. Therefore, for $K=32$ and $N=4$, the computational advantage is a factor of 256. Clearly this is a significant improvement and this increases for larger $K$.

\subsection{Experiments}

Many simulation and some real experiments have been done. Some results are presented here. Fig. 1 shows the results of a simulation experiment using a Gaussian PSF where the blur parameter sigma was about 2.8 pixels at the center and 
decreased linearly to about 0.2 pixels with distance near the edges. Image size was $469 \times 188$.

Fig. 2 shows the results of experiments on real image data. A slanted planar object with printed characters was imaged by a digital camera. The SV-PSF was estimated to be a Gaussian with $\sigma$ decreasing linearly from about 2.5 pixels at the left edge to 0.5 pixels near the center and then increasing back to 2.5 pixels near the right edge. Image size was $640 \times 240$. All results are quite satisfactory.

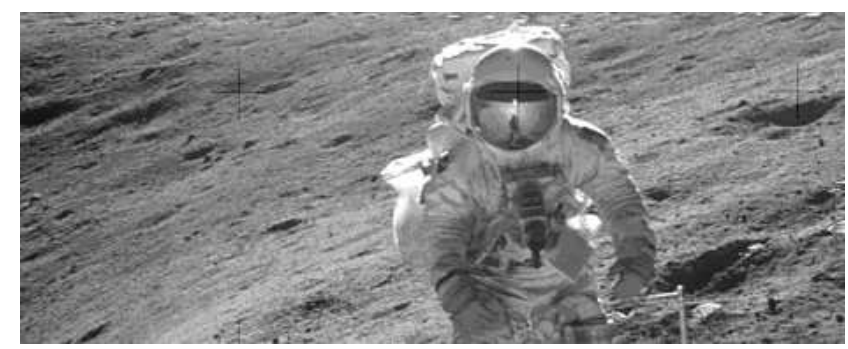

(a) Input focused image (source: NASA website)

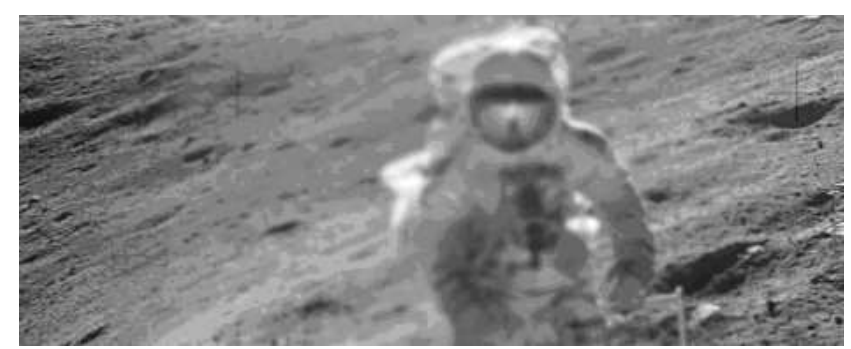

(b) Computed shift-variant Gaussian blurred image

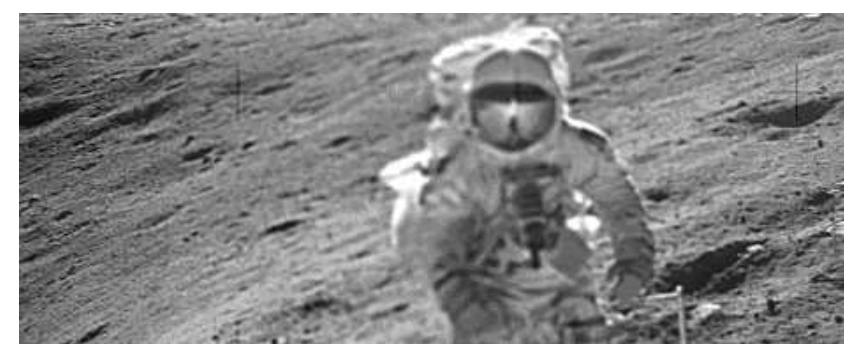

(c) Result of shift-variant image restoration by RT

Fig. 1. Results of Simulation Experiments

\section{CONCLUSION}

We have presented the basic theory, algorithm, computational complexity analysis, and experimental results for a new shiftvariant image restoration approach based on RT. The new approach is completely localized and has significant theoretical and computational advantages in image deblurring, shiftvariant signal filtering, and solving integral equations. Experimental results indicate that it is useful in practical applications. Further investigation is underway on noise sensitivity analysis and relative performance in comparison with other state-of-the-art approaches.



(a) Real image of a horizontally slanted planar object with shift-variant blur

(b) Result of shift-variant image restoration by RT

Fig. 2. Results of Real Experiments

\section{REFERENCES}

[1] J. H. Money, "Variational Methods for Image Deblurring and Discretized Picard's Method", Ph. D. Thesis, Dept. of Mathematics, University of Kentucky, 2006.

[2] M. Subbarao and G. Surya, "Depth from defocus: A spatial domain approach," International Journal of Computer Vision, vol. 13, no. 3, pp. 271-294, 1994.

[3] T. Wei M. Subbarao and G. Surya, "Focused image recovery from two defocused images recorded with different camera settings," IEEE Transactions on Image Processing, vol. 4, no. 12, pp. 1613-1628, 1995.

[4] J.G. Nagy P. C. Hansen and D. P. O'Leary, Deblurring Images: Matrices, Spectra, and Filtering, SIAM, 2006, ISBN 0-89871-618-7.

[5] J. G. Nagy and D. P. O'Leary, "Restoring images degraded by spatially-variant blur," SIAM Journal on Scientific Computing, pp. 1063-1082, 1998.

[6] A. K. Jain, Fundamentals of Digital Image Processing, Prentice-Hall, Inc., 1989, ISBN 0-13-336165-9.

[7] W. K. Pratt, Digital Image Processing, WileyInterscience, 2007, ISBN 978-0-471-76777-0.

[8] M. Subbarao, Rao Transforms: A New Approach to Integral and Differential Equations, Self-published book, 2005, 2007, 130 pages, U.S. Copyright Registration No. TX 6-591-547, June 29, 2007, see http://www.integralresearch.net.

[9] M. Subbarao, U.S. Patent Application No. 11/235724, filed on 9/26/05, and U.S. Patent Application No. 11/242191, filed on $10 / 03 / 2005$, see http://www.integralresearch.net. 